\title{
Hyperbaric Oxygen Therapy (HBO) for the Treatment of an Epidural Abscess in the Posterior Fossa in an 8-Month-Old Infant
}

\author{
H. Baechlia $\quad$ J. Schmutz ${ }^{b} \quad$ J.-M. Mayr ${ }^{c}$ \\ ${ }^{a}$ Neurosurgical Department and Children's University Hospital, b HBO Centre, University Hospital, and \\ 'Pediatric Surgical Department, Children's University Hospital, Basel, Switzerland
}

\section{Key Words}

Epidural abscess $\cdot$ Osteomyelitis $\cdot$ Hyperbaric oxygen therapy, infant

\begin{abstract}
Epidural abscesses in children are extremely rare, especially in the posterior fossa. In some cases antibiotic therapy and surgical drainage are insufficient for complete healing. We present the case of an 8-month-old boy who developed an epidural abscess in the posterior fossa after repeated surgical procedures for retrocerebellar arachnoid cysts and hydrocephalus. We decided to use adjuvant hyperbaric oxygen therapy (HBO) to avoid removal of the bone and the existing ventriculoperitoneal shunt. In this way osteomyelitis, potentially leading to bone removal and shunt infection, could be prevented. $\mathrm{HBO}$ is a relatively safe, noninvasive and cost-effective therapy to improve healing of chronic and deep-seated wound infections. To our knowledge HBO has never been used before in such a young child in neurosurgery. Multidisciplinary management is recommended to optimize treatment.

Copyright $\odot 2008$ S. Karger AG, Basel
\end{abstract}

\section{Introduction}

Intracranial epidural abscesses are extremely rare in children, especially in the very young. They mostly present with subperiostal suppuration of the calvaria or skull base as a result of sinusitis, trauma or surgical procedures [1]. Hematogenous spreading to the extradural space is rare [2]. Repeated extensive surgical procedures are timeconsuming and very burdensome and carry a high rate of complications, especially in infants. Early antibiotic therapy combined with surgical drainage of the abscess might decrease the high mortality rate [3, 4] but nevertheless this situation is a challenging problem which requires a multimodal approach. Aggressive surgical debridement with consecutive loss of bone tissue should be avoided, especially in very young children. Hyperbaric oxygen therapy (HBO) offers an additional option in the combined treatment of brain abscesses in children [5]. Today, HBO is increasingly used in difficult and complex wound healing problems but it is less known in neurosurgical patients, especially in infants. HBO is currently used in the primary therapy of arterial gas embolism, decompression sickness and severe carbon monoxide poisoning. It is also useful as adjunctive therapy in compartment syndrome, complex wound problems, osteoradionecrosis, radiationinduced soft tissue injury, refractory osteomyelitis and acute traumatic ischemic injuries [6]. The first hyperbaric chamber was built in 1662 by Henshaw [7] and in the ear-

Dr. Heidi Bächli

Neurosurgical Department University Hospital Basel Spitalstr. 21

CH-4031 Basel (Switzerland)

Tel. +41 61265 2525, Fax +4161265 7138, E-Mail hbaechli@uhbs.ch 


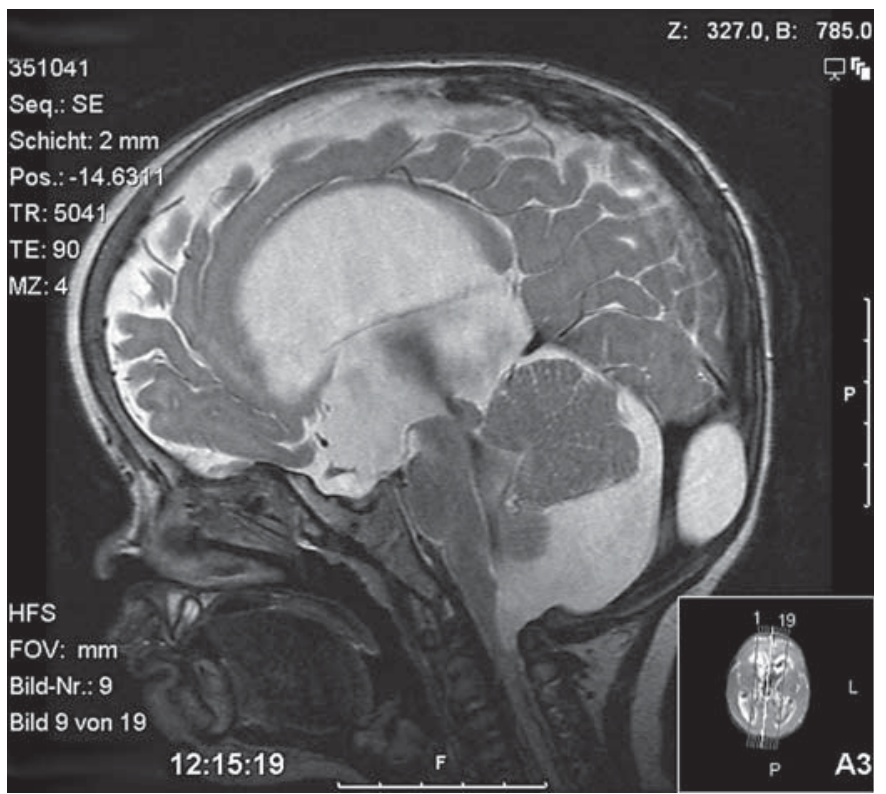

Fig. 1. Eight-month-old boy. Sagittal $\mathrm{T}_{2}$-weighted MRI scan showing retrocerebellar arachnoid cysts with hydrocephalus.

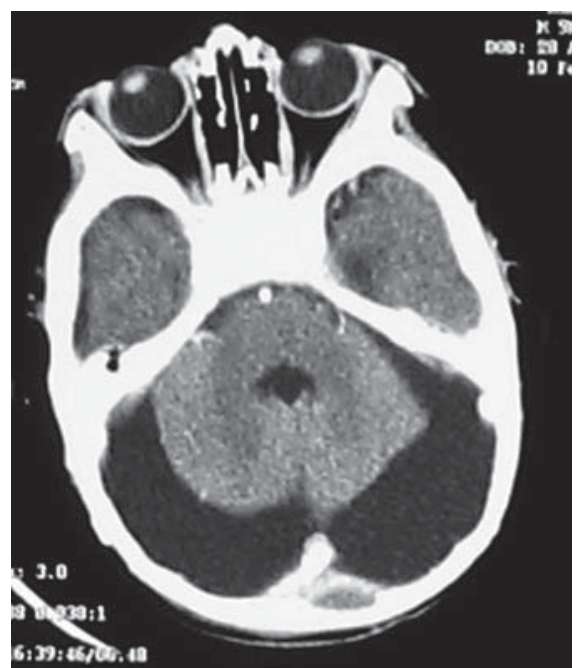

Fig. 2. CCT scan 3 weeks later with enhancing suboccipital epidural abscess.

ly 1960s anaerobic infections were first treated using $\mathrm{HBO}$ [8]. The Committee on Hyperbaric Oxygen Therapy is the international authority which regulates and controls the use of HBO. Hyperbaric chamber use in infants is limited [5]. We present a case in which an epidural abscess of an 8 -month-old infant was successfully managed with antibiotic and additional HBO therapy.

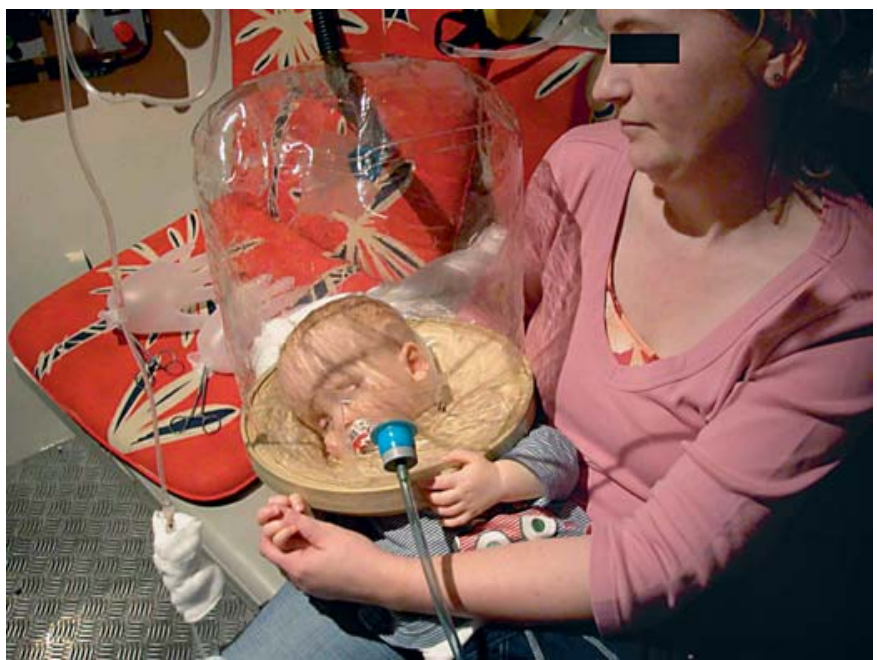

Fig. 3. Eight-month-old boy with mother during HBO.

\section{Case Report}

We describe an 8-month-old boy who suffered from macrocephaly due to retrocerebellar arachnoid cysts with consequent hydrocephalus (fig. 1). In a first step a suboccipital craniotomy with cyst wall excision was carried out to avoid shunting. Subsequently the infant developed a cutaneous cerebrospinal fluid fistula, which did not respond to conservative therapy, repeated punctures or acetazolamide. After 10 days the craniotomy was revised, the dural fistula closed with fascia lata and an external ventricular drainage was inserted. Because of persistent hydrocephalus the external drainage had to be removed and 10 days later a programmable ventriculoperitoneal shunt $\left(\right.$ Hakim $^{\mathrm{TM}}$ programmable valve with Siphonguard ${ }^{\mathrm{TM}}$ device and reservoir, Codman) was inserted. The patient recovered well and could be discharged home. Ten days later the mother observed a small wound dehiscence and a surgical revision of the wound was carried out. Swabs for bacterial examination were taken and an antibiotic therapy with oral clindamycin was started. Staphylococcus aureus was cultured as the pathogenic germ. One month later a new swelling and redness of the wound with a fluid collection was noted.

Blood parameters showed only an elevated C-reactive protein level of $57 \mathrm{mg} / \mathrm{l}$ (normal $<10 \mathrm{mg} / \mathrm{l}$ ) and the CT scan revealed an epidural abscess in the suboccipital area (fig. 2). The abscess was immediately surgically drained, and bacterial cultures were obtained. Cerebrospinal fluid puncture analysis was normal. The antibiotic treatment was now extended to include both ceftriaxone and clindamycin intravenously. To ameliorate dermal wound healing and to prevent bone removal in the posterior fossa together with explantation of the ventriculoperitoneal shunt, we decided to combine the treatment with HBO. Before this therapy was started, the child needed a paracentesis of both middle ears because of 
inadequate pressure equalization. HBO therapy was then administered in the presence of the mother once daily in a multiplace chamber with $100 \%$ oxygen inhalation for $2 \times 45 \mathrm{~min}$ with a pressure of 2.4 ATA (fig. 3). A total of 20 sessions were applied. Six weeks later the MRI showed regression of the hydrocephalus and retrocerebellar cysts as well as the disappearance of the epidural abscess. The wound was no longer inflamed. The infant recovered completely. No side effects of the hyperbaric therapy were observed. The evaluation 6 months later showed no recurrence of the epidural abscess, osteomyelitis or shunt infection.

\section{Discussion}

In pediatric neurosurgical centers about 1 case of epidural abscess per year will be expected for hospitalization [9]. In early stages or selected cases conservative treatment may be justified $[1,10,11]$. Indications for neurosurgical drainage are space-occupying mass effects on CT or MRI or enlarging abscesses despite antibiotic therapy. Diagnostic procedures for bacteriological identification can be necessary. The medical treatment should begin as early as possible and intravenous antibiotics are usually applied for 6-8 weeks, followed by oral antibiotics for another 4-6 weeks in cases of brain abscess [12]. In our case the infant was treated with ceftriaxone and clindamycin intravenously for 6 weeks, followed by 6 weeks of oral medication with clindamycin. The treatment in our patient was additionally complicated by the presence of a ventriculoperitoneal shunt and the critically vascularized fascia lata flaps. Under these conditions, removal of the bone flap in the posterior fossa and the removal of the shunt seemed to be unavoidable, which is not harmless in a child of this age. In our case we could not be sure that the change of antibiotics alone would be sufficient to resolve the infection. Vacuum applicators were not considered because they are only effective for superficial wounds. So we decided to use additional HBO, which is a wellknown method for the healing of deep-seated wounds [5, 13]. Oxygen has a direct antimicrobial effect, particularly on anaerobes. Oxygen in hypoxic tissues can lead to restoration of white blood cells and antimicrobial function $[6,13,14]$.

Further advantages are acceleration of wound healing which leads to a reduction of surgical procedures including its related risks, especially in the very young. Another important point in favor of $\mathrm{HBO}$ is the lower cost caused by the reduction of extensive medical and surgical care. The utilization of $\mathrm{HBO}$ in infants is only restricted to case reports in other specialities for instance for hepatic artery thrombosis following liver transplantation [15].

Hyperbaric Oxygen Therapy in Infants
An average 90 -min $\mathrm{HBO}$ treatment in the USA costs between USD 300 and 400. For example a therapy for osteoradionecrosis or problem wounds needs $30-40$ sessions and the costs range between USD 9,000 and 16,000, this means savings of USD 96,000 compared with conventional therapy [6]. In our case 20 sessions of $2 \times 45$ min were needed for a total cost of USD 4,570 (EUR $3,604)$.

The average number of treatments for chronic wounds is 30 , its utilization review is recommended after 20 treatments $[16,17]$. Although HBO is relatively safe, some risks and complications, especially in children, should be considered. Most side effects are related to pressure/volume changes. Possible complications include barotraumaticlesions (middle ear, nasal sinuses, inner ear, lung, teeth), oxygen toxicity (central nervous system irritability, decreased visual fields, nausea, tinnitus, dizziness and generalized convulsions, lung lesions), claustrophobia and ocular effects (reversible myopia, cataract formation after more than 150 consecutive sessions) $[6,13,18]$. Absolute contraindications for $\mathrm{HBO}$ are an untreated pneumothorax and concomitant chemotherapy with doxorubicin or cisplatin. In children younger than 4 years of age, inadequate pressure equalization in the ears might pose a problem. As in our case, a paracentesis can be performed. If a child is unwilling or afraid to enter the hyperbaric chamber, a family member should accompany the child. In our case the mother accompanied the child in all of the 20 sessions. $\mathrm{HBO}$ is increasingly used in different medical areas and is an established therapy in life-threatening and chronic diseases. In the last years there has been an increasing number of reports of children treated successfully with hyperbaric oxygen [13] but there is only 1 publication about its use in pediatric neurosurgery [5]. All of the children were older than 10 years. In our clinic we mainly have experience with adults. We chose this therapy because it is noninvasive, psychologically less traumatic for the child and costs less than multiple reoperations with consecutive long hospitalizations.

Multidisciplinary management is recommended for such challenging therapies; in our case there was a collaboration between neurosurgeons, pediatric surgeons, pediatricians, anesthesiologists, intensive care, ENT and $\mathrm{HBO}$ specialists. Application of $\mathrm{HBO}$ in neurosurgery for children is innovative and should be considered in complicated and deep-seated wound infections. To our knowledge $\mathrm{HBO}$ has never been used before in such a young child in neurosurgery. Multicenter studies are recommended to confirm the significance of HBO therapy in pediatric neurosurgery.

Pediatr Neurosurg 2008;44:239-242 


\section{References}

1 Heran NS, Steinbok P, Cochrane DD: Conservative neurosurgical management of intracranial epidural abscesses in children. Neurosurgery 2003;53:893-898.

-2 Sharif HS, Ibrahim A: Intracranial epidural abscess. Br J Radiol 1982;55:81-84.

-3 Khanna RK, Malik GM, RockJP, Rosenblum ML: Spinal epidural abscess: evaluation of factors influencing outcome. Neurosurgery 1996;39:958-964.

-4 Tang HJ, Lin HJ, Liu YC, Li CM: Spinal epidural abscess - experience with 46 patients and evaluation of prognostic factors. J Infect 2002;45:76-81.

5 Kurschel S, Mohia A, Weigl V, Eder HG: Hyperbaric oxygen therapy for the treatment of brain abscess in children. Childs Nerv Syst 2006;22:38-42.

6 Tibbles PM, Edelsberg JS: Hyperbaric oxygen therapy. N Engl J Med 1996;334:16421648 .
7 Henshaw N: Aero-Chalinos. Dublin, Dancer, 1664.

8 Brummelkamp WH, Hogenijk WH, Boerema I: Treatment of anaerobic infections (clostridial myositis) by drenching the tissue with oxygen under high atmospheric pressure. Surgery 1961;49:299-302.

9 Albright AL, Pollack IF, Adelson PD: Principles and Practice of Pediatric Neurosurgery. New York, Thieme, 1999, pp $1187-$ 1201.

10 Greenberg MS: Handbook of Neurosurgery, ed 5. New York, Thieme, 2001, pp 217-223.

$>11$ Leys D, Destee A, Petit H, Warot P: Management of subdural intracranial empyemas should not always require surgery. J Neurol Neurosurg Psychiatry 1986;49:635-639.

12 Mathisen GE, Johnson JP: Brain abscess. Clin Infect Dis 1997;25:763-779.

13 Waisman D, Shupak A, Weisz G, Melamed Y: Hyperbaric oxygen therapy in the pediatric patient: the experience of the Israel Naval Medical Institute. Pediatrics 1998;102:E53.
14 Meltzer T, Myers B: The effect of hyperbaric oxygen on the bursting strength and rate of vascularization of skin wounds in the rat. Am Surg 1986;52:659-662.

15 Grover I, Conley L, Alzate G, Lavine J, Van Hoesen K, Khanna A: Hyperbaric oxygen therapy for hepatic artery thrombosis following liver transplantation: current concepts. Pediatr Transplant 2006;10:234-239.

16 Gill AL, Bell CAN: Hyperbaric oxygen: its uses, mechanisms of action and outcomes. QJM 2004:97:385-395.

17 Hampson NB (ed): Hyperbaric Oxygen Therapy: 1999 Committee Report. Kensington, Undersea and Hyperbaric Medical Society, 1999.

18 Plafki C, Peters P, Almeling M, Welslau W, Busch R: Complications and side effects of hyperbaric oxygen therapy. Aviat Space Environ Med 2000;71:119-124. 